\title{
Eficiência dos testes cromáticos de comparação na discromatopsia hereditária: relato de casos
}

\author{
Efficiency of color vision tests in hereditary dyschromatopsia: case report
}

\author{
Luciene Chaves Fernandes ${ }^{1}$ \\ Lúcia Carvalho de Ventura Urbano ${ }^{2}$
}

Trabalho realizado em clínica particular das autoras.

${ }^{1}$ Coordenadora do Serviço de Visão Subnormal do Hospital São Geraldo. Doutora em Oftalmologia pela Faculdade de Medicina da Universidade Federal de Minas Gerais - UFMG - Belo Horizonte (MG) - Brasil.

${ }^{2}$ Professora Adjunta IV do Departamento de Oftalmologia-Otorrinolaringologia da Faculdade de Medicina da Universidade Federal de Minas Gerais e doutora, Livre-Docente em Oftalmologia pela Faculdade de Medicina da UFMG - Belo Horizonte (MG) - Brasil.

Endereço para correspondência: Luciene Chaves Fernandes. Rua Padre Rolim, 515 - Belo Horizonte (MG) CEP 30130-090

E-mail: lucienef@uai.com.br

Recebido para publicação em 13.02.2007

Última versão recebida em 18.05.2008

Aprovação em 23.05.2008

\begin{tabular}{|c|}
\hline RESUMO \\
\hline As autoras relatam dois casos de discromatopsia hereditária e discutem a \\
eficiência dos testes cromáticos no diagnóstico de uma discromatopsia. \\
Os pacientes foram reprovados em diferentes concursos públicos federais \\
por apresentarem diagnóstico de discromatopsia hereditária pelo teste de \\
Ishihara. Submeteram-se a exame oftalmológico, com resultados dentro da \\
normalidade. Procuraram novo parecer para melhor caracterização da sua \\
discromatopsia. Não havia sintomas relacionados à deficiência. Os testes \\
PanelD15 simples, D28 de Roth mostraram-senormais e o D15 dessaturado \\
confirmou deutanomalia no caso 1. No segundo caso os testes de compa- \\
ração mostraram alterações sem significado cromático. O diagnóstico de \\
uma discromatopsia é muito complexo. As autoras destacama importância \\
de realização de testes de visão cromática complementares ao teste de \\
Ishihara para diagnóstico de uma discromatopsia.
\end{tabular}

Descritores: Percepção de cores; Testes de percepção de cores; Testes visuais; Defeitos da visão cromática; Humano; Masculino, Adulto; Relatos de casos [Tipo de publicação]

\section{INTRODUÇÃO}

O conhecimento e caracterização dos distúrbios da visão cromática têm se tornado de importância crescente pela necessidade da percepção correta das cores em atividades artísticas, profissionais, industriais ou mesmo científicas.

Newton (1664), citado por Brindley (1957), ao interpor um prisma no trajeto dos raios de luz que passavam pela fresta de sua janela descobriu por acaso o espectro visível ao olho humano. Observou a imagem refratada pela refringência desigual dos raios luminosos decomposta nas sete cores primárias: vermelho, laranja, amarelo, verde, azul-verde, azul e violeta. O comprimento deste feixe luminoso estende-se de 380 a 780 nanômetros $(\mathrm{nm})^{(1)}$.

Dalton (1798) foi o primeiro a descrever sua alteração, admitindo que o seu vítreo estaria tingido de azul, impossibilitando a passagem da luz vermelha. A partir daí passou a ser chamada de Daltonismo a percepção anormal das $\operatorname{cores}^{(2)}$. Hoje o termo preferido é discromatopsia (dis=distúrbio; cromos $=$ cor; opsis $=$ olho) que significa todo e qualquer distúrbio cromático ${ }^{(3)}$.

Segundo Wright (1957) do ponto de vista cromático os indivíduos podem ser classificados como tricromata (normal e anormal), dicromata, acromata ou monocromata ${ }^{(3)}$. Os tricromatas normais, em luminosidade habitual, percebem todo o espectro visível ao olho humano, desde o azul em torno de $380 \mathrm{~nm}$, passando pelo verde em torno de $500 \mathrm{~nm}$ até o vermelho em $780 \mathrm{~nm}$. Não identificam o ultravioleta (abaixo de $380 \mathrm{~nm}$ ), nem o infravermelho (acima de $780 \mathrm{~nm}$ ). Já os tricromatas anormais percebem deficitariamente uma das três cores básicas. Para vê-la corretamente é necessário aumentar 
uma das três variáveis da luz monocromática: saturação, tonalidade e brilho. É a protanomalia no déficit para o vermelho; a deutanomalia, para o verde; a tritanomalia para o azul e a tetartanomalia para o amarelo. O dicromata não percebe uma das três cores, mesmo modificando uma das três variáveis: é o protanope, deutanope, tritanope ou tetartanope. $\mathrm{O}$ acromata ou monocromata não percebe nenhuma das três cores básicas identificando, somente brilhos diferentes das mesmas.

As discromatopsias podem ser: congênitas (melhor chamadas de hereditárias) ou adquiridas. As formas hereditárias protan e deutan são recessivas ligadas ao cromossomo $\mathrm{X}$; o distúrbio tritan é autossômico dominante e a acromatopsia autossômica recessiva. Já as formas adquiridas, sem componente genético são devidas a doenças sistêmicas ou oculares.

O Atlas de Munsell (1942) tem 1226 nuances cromáticas iniciando-se no vermelho púrpura e fechando o círculo no azul violeta $^{(4)}$. O Teste de Ishihara (para discromatopsia hereditária), idealizado anteriormente à descrição do Atlas, teve as pranchas construídas com pigmentação em torno de $565 \lambda$ (ondas longas L) considerado ponto neutro ou eixo de confusão do protan. Nele há visualização deficitária pelo protanômalo ou nenhuma pelo protanope. A pesquisa do deutan é feita nas pranchas com pigmentação em torno de $543 \lambda$ (aonde se situam as ondas intermediárias $\mathrm{M}$ ), vistas deficitariamente pelo deuteranômalo e ausente no deutanope.

A primeira edição do Teste de Ishihara foi publicada em Tokyo (1917). A edição de 1996 constando de 24 pranchas, com números ou estradas sinuosas (para crianças e iletrados) tem pigmentação cuidadosamente escolhida para cair dentro das áreas aonde os defeitos cromáticos acentuam-se. Os círculos do fundo variam em tonalidade, saturação e brilho, com diâmetros de um a cinco milímetros cada. Classifica as alterações cromáticas em moderada (protanomalia ou deutanomalia) e forte (protanopia ou deutanopia); além de acromatopsia ${ }^{(5)}$.

Os testes de comparação (Panel D15, D15 dessaturado e D28 de Roth) indicados nas discromatopsias adquiridas são construídos abrangendo todo o círculo de Munsell, uma vez que o processo é resultante de uma alteração em intensidade variável nos cones retinianos. Respeita-se, no entanto, os eixos definidos para caracterizar as três patologias cromáticas: protan, deutan, $\operatorname{tritan}^{(6-7)}$.

Panel D 15, descrito em detalhes por Linsksz (1964), consta de um peão $(\mathrm{P})$ de referência e 15 pastilhas coloridas numeradas no verso, com saturação cinco e brilho cinco. Para o exame as pastilhas são misturadas numa mesa de fundo preto e o paciente a partir do $\mathrm{P}$ coloca ao seu lado a que lhe pareça mais semelhante; sucessivamente ordenando as 15 . Anota-se o número contido no verso e faz-se o traçado, unindo os pontos ${ }^{(7)}$.

Panel D 15 dessaturado - Lanthony (1984), admitiu que a saturação é o primeiro fator da sensação colorida a se degradar. Assim, as pastilhas do Panel D 15 com saturação cinco e brilho cinco ele dessaturou para dois e aumentou o brilho para oito. Tornou, portanto, as 15 pastilhas do D15 dessaturado mais claras e brilhantes. Os resultados são transcritos para um gráfico igual ao do Panel D15(8).
D 28 de Roth (1966) o autor separou uma pastilha de cada três do Farnsworth 100 Hue mantendo no verso das selecionadas o número original. A saturação cinco e o brilho cinco foram mantidos. A ordenação feita pelo paciente é anotada e transcrita para o gráfico de forma $\operatorname{circular}^{(7)}$.

Tem sido demonstrado ao longo dos anos que um único teste de visão de cores não é suficiente para um diagnóstico conclusivo de uma discromatopsia, tornando necessária a realização de vários exames.

As autoras discutem a partir de dois casos de discromatopsia hereditária a resposta aos diferentes testes cromáticos.

\section{RELATO DE CASOS}

\section{Caso 1}

ORC, masculino, 27 anos, leucodérmico. Aprovado em primeira etapa de concurso público para o cargo de agente de Polícia Federal, realizou exame oftalmológico, que se mostrou sem anormalidades. Pelo teste de Ishihara diagnosticouse discromatopsia hereditária, o que o reprovou na avaliação clínica. Procurou novo parecer para melhor caracterização de sua discromatopsia, desconhecida até então, pelo mesmo.

Ao exame apresentava:

- Acuidade visual OD=20/20 (1,0) com -0,50 - 1,50 x $160^{\circ}$ $\mathrm{OE}=20 / 20(1,0)$ com $-0,50-1,25 \times 10^{\circ}$;

- Exame oftalmológico sem outras alterações;

- Testes cromáticos:

Iniciou-se o exame com as Tábuas de Ishihara, edição 1996. Foram mostradas, monocularmente, as 24 pranchas de números, em ângulo reto com a linha de visão do examinado, a aproximadamente 40 centímetros do olho, uma após a outra, com intervalos de três a cinco segundos. O resultado do teste foi semelhante em ambos os olhos, diagnosticando deutanomalia bilateral (Tabela 1).

Nos testes de comparação (Panel D15, D15 dessaturado e D28 de Roth), também realizados monocularmente, conforme a normatização dos exames, obteve-se os seguintes resultados:

Panel D 15: traçado normal em ambos os olhos.

D 15 dessaturado: traçado sugestivo de deutanomalia (Gráfico 1$)$.

D 28 de Roth: traçado normal em ambos os olhos.

\section{Caso 2}

JEMBF, masculino, 15 anos, leucodérmico, submeteu-se a concurso público para admissão em Colégio Naval no Rio de Janeiro. Reprovado por apresentar discromatopsia hereditária diagnosticada através do teste de Ishihara procurou novo parecer oftalmológico.

Apresentava:

- AV OD=20/20 sem correção

$\mathrm{OE}=20 / 20$ sem correção;

- Exame oftalmológico sem alterações;

- Testes cromáticos: Como no caso 1 , realizou todos os 


\begin{tabular}{|c|c|c|c|c|c|c|c|}
\hline Prancha & $\begin{array}{c}\text { Resposta } \\
\text { normal }\end{array}$ & \multicolumn{4}{|c|}{$\begin{array}{l}\text { Discromatopsia } \\
\text { vermelho-verde }\end{array}$} & $\begin{array}{c}\text { Caso } \\
1\end{array}$ & $\begin{array}{c}\text { Caso } \\
2\end{array}$ \\
\hline 1 & 12 & \multicolumn{4}{|c|}{12} & 12 & 12 \\
\hline 2 & 8 & \multicolumn{4}{|c|}{3} & 3 & 3 \\
\hline 3 & 29 & \multicolumn{4}{|c|}{70} & 79 & 70 \\
\hline 4 & 5 & \multicolumn{4}{|c|}{2} & 2 & $\mathrm{x}$ \\
\hline 5 & 3 & \multicolumn{4}{|c|}{5} & 5 & $\mathrm{X}$ \\
\hline 6 & 15 & \multicolumn{4}{|c|}{17} & 17 & 15 \\
\hline 7 & 74 & \multicolumn{4}{|c|}{21} & 21 & 21 \\
\hline 8 & 6 & \multicolumn{4}{|c|}{$\mathrm{x}$} & $x$ & $\mathrm{x}$ \\
\hline 9 & 45 & \multicolumn{4}{|c|}{$x$} & $x$ & $x$ \\
\hline 10 & 5 & \multicolumn{4}{|c|}{$x$} & $x$ & $x$ \\
\hline 11 & 7 & \multicolumn{4}{|c|}{$X$} & $\mathrm{X}$ & $\mathrm{X}$ \\
\hline 12 & 16 & \multicolumn{4}{|c|}{$X$} & $x$ & $x$ \\
\hline 13 & 73 & \multicolumn{4}{|c|}{$\mathrm{X}$} & $x$ & $\mathrm{X}$ \\
\hline 14 & $\mathrm{x}$ & \multicolumn{4}{|c|}{5} & $x$ & $\mathrm{X}$ \\
\hline \multirow[t]{3}{*}{15} & $\mathrm{x}$ & \multicolumn{4}{|c|}{45} & $\mathrm{X}$ & $\mathrm{x}$ \\
\hline & & \multicolumn{4}{|c|}{ Protan } & & \\
\hline & & $\bar{F}$ & $\bar{M}$ & $\bar{F}$ & $M$ & & \\
\hline 16 & 26 & 6 & (2) 6 & 2 & $2(6)$ & $2(8)$ & 26 \\
\hline 17 & 42 & 2 & $(4) 2$ & 4 & $4(2)$ & $4(2)$ & 42 \\
\hline
\end{tabular}

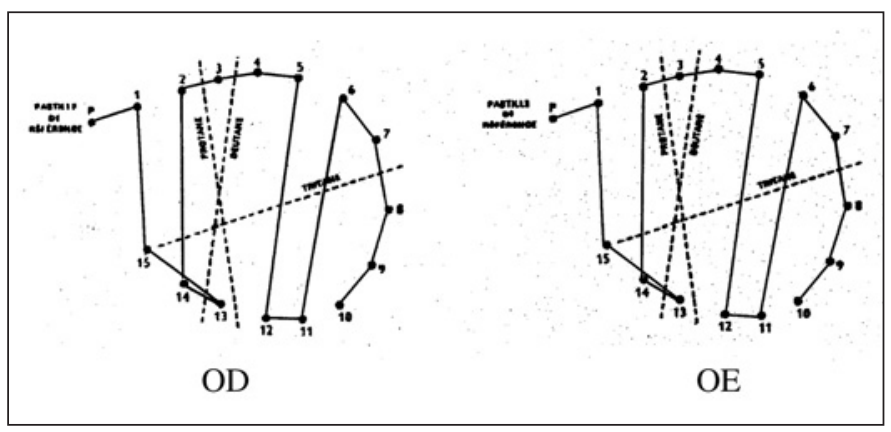

Gráfico 1 - Resultado do teste Panel D 15 dessaturado do caso 1

testes em uso de lentes corretoras incolores, com iluminação ambiente acrescida de foco incandescente de 60 watts.

- Teste de Ishihara: oito respostas erradas naquelas que identificaria alterações em protan ou deutan. Naquelas que classifica a discromatopsia em moderada ou forte, identificou todas corretamente. (Tabela 1)

- Panel D 15: uma inversão periférica, sem significado cromático (Gráfico 2).

- D 15 dessaturado: quatro inversões periféricas, sem significado cromático (Gráfico 3).

- D28 Roth: duas inversões periféricas, sem significado cromático (Gráfico 4).

\section{DISCUSS ÃO}

Os testes de visão de cores têm como objetivo classificar o indivíduo como normal e anormal para a percepção de cores e

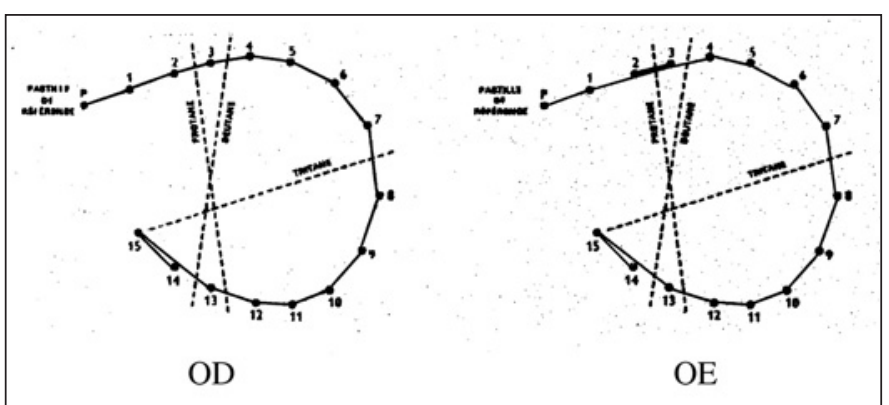

Gráfico 2 - Resultado do teste Panel D 15 do caso 2

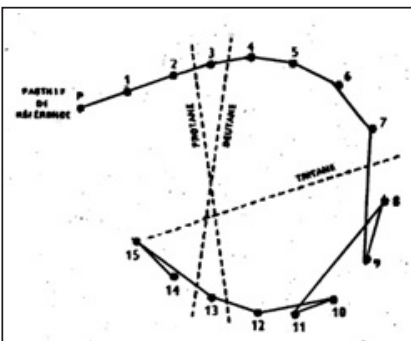

OD

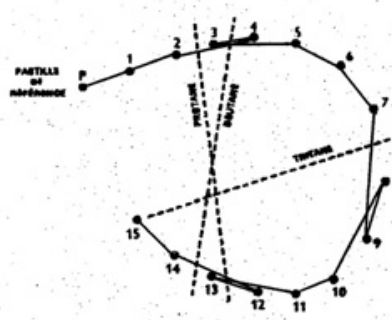

$\mathrm{OE}$
Gráfico 3 - Resultado do teste Panel D 15 dessaturado do caso 2

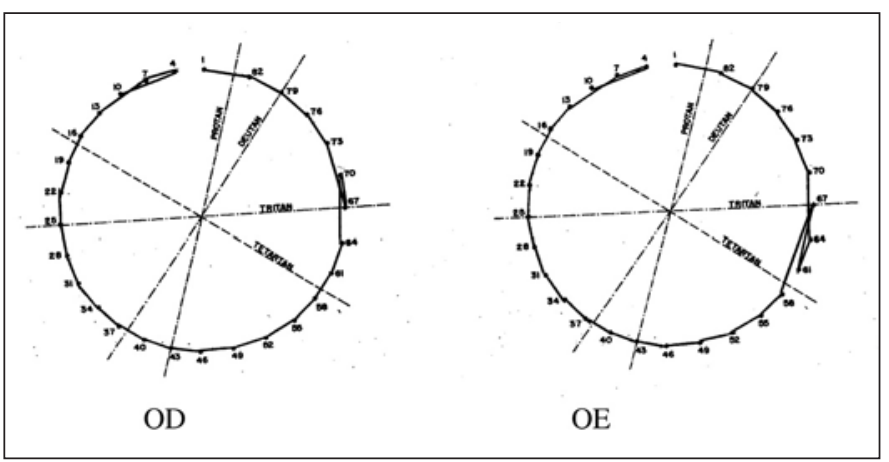

Gráfico 4 - Resultado do teste D 28 de Roth do caso 2

os diferentes graus da discromatopsia: leve, moderada e forte. Todos os testes cromáticos apresentam limitações: degradação e modificação dos tons pelo tempo, variabilidade da iluminação e mesmo informação do paciente.

As Tábuas de Ishihara (primeira edição em 1917) têm sido reeditadas ao longo dos anos. Os cartões de confusão das cores foram cuidadosamente escolhidos para cair nas áreas aonde os defeitos cromáticos acentuam-se. É o mais usado teste de visão de cores e considerado padrão para uma rápida identificação de uma discromatopsia hereditária no eixo vermelho-verde ${ }^{(9-10)}$. Cada prancha tem sensibilidade e especificidade entre 85 a $95 \%$ e o exame completo muito próximo a $100 \%$. Às vezes, falha no diagnóstico de uma discromatopsia moderada ou detecta uma, em indivíduo normal, que apresenta apenas uma discriminação baixa para cores. No rastreamento de uma população geral para 
discromatopsia hereditária, pode-se aplicar apenas o teste de Ishihara, já que nesta forma há uma preponderância de mais de 99\% de alterações vermelho-verde (sendo a deutanomalia a mais freqüente). No diagnóstico de certeza de uma discromatopsia, recomenda-se a combinação de vários testes.

As instruções editoriais das Tábuas de Ishihara consideram tricromata normal para até três erros; acima de seis erros, o indivíduo seria portador de uma discromatopsia. Entre três e seis erros o diagnóstico deve ser de incerteza, necessitando de testes cromáticos complementares, para confirmação. Alguns autores diagnosticam como normal, numa seleção ocupacional, o paciente que comete até três erros nas Tábuas de Ishihara ${ }^{(11)}$.

Birch (1997) ao examinar 401 homens com discromatopsia hereditária concluiu que com um mínimo de três erros identifica-se $98,7 \%$ dos distúrbios cromáticos no eixo vermelho-verde. No restante $1,3 \%$ de tricromatas anormais (todos deutanômalos) o teste mostrou-se normal. Ele destaca ainda, maior sensibilidade no deutan ${ }^{(10)}$.

Nas discromatopsias a saturação é o primeiro fator da sensação colorida a se degradar. As cores tornam-se pálidas, antes de deixarem de ser percebidas. Segundo Lanthony (1984), o D15 dessaturado detecta formas leves de distúrbio cromático, que escapam ao Panel D15, quando tricromatas normais e anormais podem apresentar resultado normal ${ }^{(8)}$. Alguns autores citam o D15 dessaturado como o teste cromático mais sensível para diagnóstico de formas atípicas e adquiridas de uma discromatopsia ${ }^{(12)}$.

Não há normas pré-estabelecidas relacionadas à visão cromática para os diversos concursos públicos, de forma que cada edital apresenta as suas exigências. As autoras sugerem uma padronização das discromatopsias para as diversas profissões, possibilitando uma melhor orientação profissional aos discromatas.

No caso 1 o teste de Ishihara mostrou mais de seis erros, o Panel D15 e o D28 de Roth foram normais e o D 15 dessaturado confirmou deutanomalia. Desta forma admitimos tratar-se de uma forma leve ou atípica de discromatopsia.

No segundo caso, o paciente apresenta uma discromatopsia hereditária leve no eixo vermelho-verde, não sendo possível identificar se se trata de protanomalia ou deutanomalia.

O diagnóstico de uma discromatopsia é muito complexo. A discriminação cromática, mesmo quando normal, pode ser classificada em alta (acima da média), normal e baixa.
As autoras destacam a importância de realização de testes de visão cromática complementares ao Ishihara quando a caracterização do tipo e intensidade da discromatopsia se torna necessário.

\section{ABSTRACT}

The authors describe two cases of hereditary dyschromatopsia and discuss the efficiency of the color vision tests. The patients were disapproved in different federal public examinations because Ishihara's test diagnosed hereditary dyschromatopsia. Ophthalmological evaluation was normal. No symptoms related to dyschromatopsia were presented. Panels D15 and Roth D 28 were normal. Desaturated D 15 showed deuteranomaly in case one. In the second case the comparative color vision tests showed nonspecific disorder. The diagnosis of dyschromatopsia is complex. The authors recommend comparative color vision tests to complement the Ishihara test for a better understanding of the color deficiency.

Keywords: Color perception; Color perception tests; Vision tests; Color vision defects; Human; Male; Adult; Case reports [Publication type]

\section{REFERÊNCIAS}

1. Brindley GS. Human colour vision. Prog Biophys Biophys Chem. 1957;8:49-94

2. Dalton J. Extraordinary facts relating to the vision of colours: with observations Mem Proc Manchester Lit Philo Soc. 1798;5:28-45.

3. Wright WD. Colour vision. Surv Ophthalmol. 1957;2:476-93.

4. Munsell AH. Munsell color system. J Opt Soc Am. 1942;30:573-645.

5. Ishihara $\mathrm{S}$. The series of plates designed as a test for colour-deficiency. 24 plates ed. Tokyo: Kanehara; 1996. p.1-9.

6. Farnsworth D. The Farnsworth dichotomus test for color blindness - Panel D15 New York: Psychological Corporation; 1946.

7. Urbano LC. Discromatopsia: métodos de exame [tese]. Belo Horizonte: Universidade Federal de Minas Gerais; 1976.

8. Lanthony P. Étude de la saturation au cours de dischromatopsies acquises au moyen de L'album de Munsell. Ann Occult. 1984;207:741-51.

9. Dain SJ. Clinical colour vision tests. Clin Exp Optom. 2004;87(4-5): 276-93.

10. Birch J. Efficiency of the Ishihara test for identifying red-green colour deficiency. Ophthalmic Physiol Opt. 1997;17(5):403-8.

11. Margrain TH, Birch J, Owen CG. Colour vision requirements of firefighters. Occup Med (Lond). 1996;46(2):114-24.

12. Hyvarinen L, Lindstedt E. Assessment of vision in children. Stockholm: SRF Tal \& Punkt; 1981. 